\title{
Non-muscle invasive high grade urothelial carcinoma of the bladder. Which factors can influence understaging at the time of radical cystectomy?
}

\author{
Daniele Minardi, Giulio Milanese, Gianni Parri, Vito Lacetera, Giovanni Muzzonigro \\ Department of Clinic and Specialistic Sciences, Urology, Polytechnic University of the Marche Region, \\ Azienda Ospedaliero-Universitaria Ospedali Riuniti, Ancona, Italy.
}

\begin{abstract}
Summary Objective: To evaluate the main factors which influence understaging in patients with T1G3 non-muscle invasive bladder cancer (NMIBC). Materials and methods: 109 patients with T1/G3 underwent transurethral resection of bladder tumor (TURBT) and then radical cystectomy $(R C)$ with pelvic lymph nodes dissection. A number of variables were considered when evaluating the detection of understaging. We considered the patients age and gender, as well as the size, number, location and morphology of their tumor. We also considered coexistence of bladder carcinoma in situ (CIS), microscopic vascular invasion and deep lamina propria invasion. The level of experience of the surgeon was also analyzed.

Results: in RC samples muscle invasion, that is understaging, was detected in 74 (67.9\%) patients, while 35 (32.1\%) patients were appropriately staged. In these cohort of patients with high grade tumors, understaging was associated with deep lamina propria and microscopic vascular invasion, multiple tumors, tumor size $>6 \mathrm{~cm}$, tumor location (trigone and dome), presence of residual tumor; age, gender, tumor morphology, CIS associated, and experience of urological surgeon were not associated with clinical understaging. Conclusions: in our study, evaluating patients with high grade NMIBC at first TURBT, we identified some risk factors that need to be considered and that are able to increase the risk of understaging: deep lamina propria and microscopic vascular invasion, multiple tumors, tumor size $>6 \mathrm{~cm}$, tumor location (trigone and dome), presence of residual tumor. When these risk factors are present, performing an early cystectomy, and not a re-TURBT, could lower the risk of worse pathological finding due to rapid disease progression of the high grade tumors, and can prolong survival.
\end{abstract}

KEY WORDS: Bladder tumor; High grade bladder carcinoma; Non-muscle invasive bladder carcinoma; Understaging.

Submitted 7 March 2015; Accepted 29 March 2015

\section{INTRODUCTION}

Bladder cancer is a frequent malignancy of the lower urinary tract, showing an incidence of 10.1 per 100,000 for men and 2.5 per 100,000 for women (1). The majority of patients with bladder cancer at first diagnosis have non-muscle-invasive bladder tumors (NMIBC) (Tcis, Ta, and T1); but in this group also high-risk bladder cancers are included, that have a high risk of recurrence and stage progression (1).

Transurethral resection of the bladder tumor (TURBT) is the standard treatment for non-muscle-invasive cancer, by which the urologist should provide to the pathologist complete sampling of the tumor, the underlying bladder wall, and the edges of the resection (2). The pathologist should report specifically the histology type, the grade, the depth of lamina propria invasion, and the presence of muscle in the specimen (3).

The main objective of the initial TURBT is a correct staging of the tumor; while TURBT alone, with intravesical immune- or chemotherapy is the standard of care in NMIBC, muscle-invasive tumors (MIBC) ( $\geq \mathrm{T} 2$ or $\mathrm{pN1}$ ) will require radical approaches; TURBT is the key diagnostic modality to determine whether patients have bladder cancer that can be treated locally or requires more aggressive, surgical treatment (3).

In a significant number of cases the carcinoma at pathologic examination results to be understaged in specimens obtained from TURBT compared to those after radical cystectomy (RC); understaging is therefore defined as the presence of MIBC at RC; muscular invasion can more likely happen in high grade $\mathrm{BC}$ (4).

The aim of our study was to evaluate the main factors that influence disease understaging in patients with high grade NMIBC.

\section{Methods}

The study patients were identified from an existing prospective database of all patients who underwent RC at our institution between December 1998 and June 2008 (685 patients). Institutional Review Board approved the study design. 109 patients were selected from the database; they had NMIBC T1/G3 after the first TURBT; Tl tumors were subclassified into pTla (tumor invasion to the lamina propria above the level of the muscularis mucosa) and pTlb (tumors invading up to or beyond the muscularis mucosa without involving the muscularis propria) (5).

After TURBT, all the considered patients underwent RC and extended bilateral pelvic lymph nodes dissection; we did not perform a re-TURBT because in the bladder there 
was extensive and/or multiple disease, and/or residual disease. RC was performed within 6 weeks from TURBT in all patients. For the purpose of the study, only patients who did not undergo neoadjuvant chemotherapy before $\mathrm{RC}$, and those who did not receive adjuvant intravesical or systemic therapy after the TURBTs were considered. Five urological consultants and 5 urologists in training at our institution performed the TURBTs. Stage was assigned according to the American Joint Committee of Cancer (AJCC) guidelines (6); and the grade was defined according to the WHO grading system (7).

All our TURBT cases were performed under spinal anesthesia; both a 70- and 30-degree lens to evaluate the entire bladder surface fully have been used; all abnormal areas were recorded and then biopsied separately; a complete cystoscopic exam was repeated at the end of the TURBT to ensure that all tumors and abnormal areas have been resected.

Patients pathologically staged $\mathrm{Tl}$ at the time of RC were considered appropriately staged; those who resulted to have a > T2 were considered understaged.

For all the patients, we considered the following parameters: age, gender, tumor size, depth of lamina propria invasion, tumor morphology (solid vs. papillary) (8), associated carcinoma in situ, multiplicity, location of the tumor (lateral wall, trigone, dome), and microscopic vascular invasion. We also considered the surgeon's experience when performing TURBT (operations performed by experienced consultants or by registrars); all of these parameters were evaluated for detection of understaging.

\section{Statistical analysis}

The Mann- Whitney U test or Student's T-test methods were used to compare differences among categories as appropriate. The nonparametric Wilcoxon signed-rank test was used on calculated pair difference values. Survival curves were assessed using the Kaplan-Meier method and differences were calculated with the log-rank statistic. Multivariate analysis was performed with Cox proportional hazard model analyses to identify predictors of clinical understaging. Two-tailed tests were used for all comparisons and $\mathrm{p}<0.05$ was considered statistically significant. SPSS 11.0 software was used for all calculations

\section{Results}

The characteristics of the patients who took part in the study are listed in Table 1. The mean age of patients (SD) was 69.4 (11.2) years; 87 of them were males and 22 females; mean tumor diameter was $5.8+1.5 \mathrm{~cm} ; 39$ patients were staged as $\mathrm{Tla}$ and $70 \mathrm{as} \mathrm{Tlb}$; in 35 patients the tumor was solid and in 74 it was papillary; CIS was associated in 12 patients; in 32 patients the tumors were single and in 77 multiple; 63 tumors were in the lateral bladder walls, 21 in the trigone, and 25 in the bladder dome; microscopic vascular invasion was present in 65 patients; 63 operations were undertaken by consultants and 46 by registrars; in 83 patients there was residual tumor after TURBT.

Final pathologic specimens from RC revealed muscle invasion, i.e. understaging, in 74 (67.9\%) patients; therefore 35 (32.1\%) patients were appropriately staged;
Table 1.

Patients characteristics.

\begin{tabular}{|lcccc|}
\hline Characteristics & After TURBT & $\begin{array}{c}\text { Understaged } \\
\text { at RC }\end{array}$ & $\begin{array}{c}\text { Appropriately } \\
\text { staged at RC }\end{array}$ & $p$ \\
\hline n. patients & 109 & 74 & 35 & \\
\hline Mean age & $69.4+11.2$ & $70.1+9.8$ & $68.5+10.9$ & 0.619 \\
\hline Male & 87 & 64 & 23 & \\
Female & 22 & 10 & 12 & 0.282 \\
\hline Tumor size & $5.8+1.5$ & $6.8+1.9$ & $4.5+1.8$ & 0.025 \\
\hline T1a & 39 & 11 & 28 & 0.002 \\
\hline T1b & 70 & 63 & 7 & \\
\hline Tumor morphology & & & & \\
Solid & 35 & 20 & 15 & 0.081 \\
Papillary & 74 & 54 & 20 & \\
\hline Bladder carcinoma & & & & \\
in situ associated & 12 & 4 & 8 & 0.284 \\
\hline Single & 32 & 3 & 29 & 0.003 \\
Multiple & 77 & 71 & 6 & \\
\hline Location & & & & \\
Lateral wall & 63 & 38 & 25 & 0.008 \\
Trigone & 21 & 17 & 4 & \\
Dome & 25 & 19 & 6 & \\
\hline Microscopic vascular & 65 & 58 & 7 & 0.001 \\
invasion & & & & \\
\hline Surgeon's experience & & & 18 & 0.5 \\
Consultant & 63 & 45 & 17 & 0.002 \\
Registrar & 46 & 29 & 33 & \\
\hline Residual tumor & 83 & 50 & & \\
\hline
\end{tabular}

Table 2.

Multivariate analysis of factors predictors of understaging.

\begin{tabular}{|lccc|}
\hline & $\mathbf{P}$ & Relative risk & $\mathbf{9 5 \%} \mathbf{~ C l}$ \\
\hline Age & 0,210 & 1,378 & $0,7-1,7$ \\
Gender & 0,161 & 4,206 & $0,8-17,6$ \\
Depth of lamina propria invasion & 0,810 & 3,591 & $0,3-6,2$ \\
Tumor size & 0,890 & 0,899 & $0,3-1,1$ \\
Tumor morphology & 0,251 & 1,335 & $0,5-1,8$ \\
Solitary vs multiple tumor & 0,530 & 0,924 & $0,4-1,4$ \\
Ca in situ associated & 0,368 & 1,322 & $0,7-1,7$ \\
Location & 0.134 & 0.432 & $0,1-1,5$ \\
Microscopic vascular invasion & 0,001 & 1,885 & $0,7-1,5$ \\
Surgeon's experience & 0,121 & 1,320 & $0,8-2,1$ \\
Residual tumor & 0,030 & 1,474 & $0,4-1,9$ \\
\hline
\end{tabular}

nine patients had lymph nodes metastases at final pathological examination.

Understaging was associated with deep lamina propria (stage Tlb) and microscopic vascular invasion, multiple tumors, tumor size (tumors $>6 \mathrm{~cm}$ are more likely to be understaged), tumor location (tumors in the trigone and in the bladder dome are more likely to be understaged), residual tumor; age, gender, tumor morphology, bladder CIS associated did not result to be important. The type of urological surgeon (consultant or registrar) was not associated with clinical understaging at final pathology. By multivariate analysis the most important factor predictive of understaging is the presence of microscopic vascular invasion. Survival analysis (Figure 1) showed a significantly higher survival rate in those patients who were appropriately staged compared to those who were understaged at final pathology $(73,5 \%$ vs. $42 \%$; log rank $\mathrm{p}=0.001$; Chi-square $=10,560$ ). 
Figure 1.

Survival in patients understaged and appropriately staged

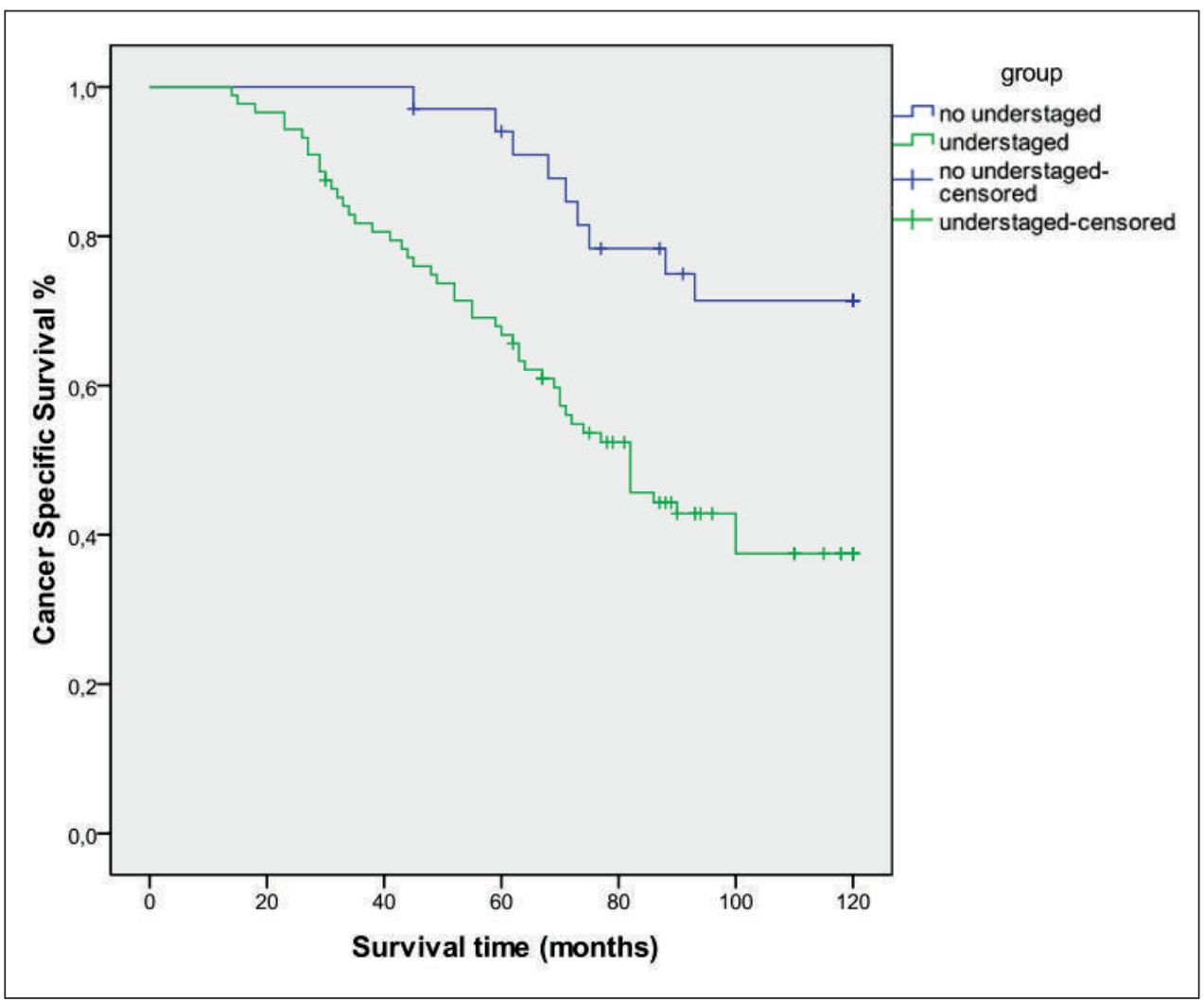

tumor than experienced surgeons; Vasdev et al. (19) did not observe that the surgeon experience was associated with the prognosis $\mathrm{TaG} 2$ tumors after restaging.

It has been observed that survival is high in patients with superficial bladder cancer after RC; survival is better in patients treated with early RC compared to those having radical surgery after recurrence of tumors (20); although $\mathrm{RC}$ is important when intravesical therapy fails, it might be too late even if the tumor remains clinically superficial. In this study we have shown that understaging is present in $67.9 \%$ of high grade NMIBC, while $32.1 \%$ of high grade NMIBC were appropriately staged; we considered a selected group of patients, who

\section{Discussion}

One of the problems of TURBT for the diagnosis and staging of $\mathrm{BC}$ is the inability to accurately identify all cases of MIBC (9). Due to the significant risk of understaging, a second TURBT is to be considered for all patients with high-grade T1 urothelial carcinoma (10); to improve the accuracy, separate biopsies from the tumor base and random bladder biopsies can provide additional prognostic informations $(3,11)$; a restaging TURBT is strongly advised for those patients diagnosed with T1 disease, where muscularis propria is not present in the initial histological specimen.

A restaging TURBT is considered for patients with highgrade $\mathrm{Ta}$ or $\mathrm{T} 1$ disease even when muscle is present in the resection specimen, due to the considerable risk of understaging, up to $70 \%$ (12-14).

Some studies have observed that a delay in doing a radical cystectomy is associated with an higher pathological stage at cystectomy; some have shown that delaying surgery more than 90 days results in a worse pathologic stage with locally advanced disease (15); others have demonstrated similar results when cystectomy is delayed of more than 12 weeks, and also a decreased survival (16). The timing of radical surgery is therefore most important.

Several studied have highlighted the importance of surgeon experience on the outcomes of TURBT. Mariappan et al. (17) observed that surgery performed by people with less than 5 years of experience was also an independent predictor of early recurrence; on the contrary, Zurkirchen et al. (18) found that trainees had less percentage of residual underwent cystectomy after the first TURBT. This was because of the presence of locally extensive and/or multiple disease and/or residual tumor, and this fact could be responsible for the high rate of understaging we have found.

In our report all the patients underwent $\mathrm{RC}$ within 6 week from TURBT, therefore a delay in performing radical surgery cannot be considered responsible for disease progression; the rate of understaging is higher compared to other studies, but we have considered a very selected cohort of patients with high grade tumors. Our finding unusual compared to the results found in the literature, and because of this we think that our findings are interesting.

We have also observed that surgeon's experience is not important in determining the rate of understaging.

In our study we have observed that there are some factors predictive of understaging: tumor size is an important factor, and tumor understaged have a significantly higher mean diameter $(6.8+1.9)$ compared to those appropriately staged $(4.5+1.8)$; tumor in the trigone are frequently understaged (probably due to early invasion of adjacent tissues), as those in the dome of the bladder (probably due to incomplete resection for technical difficulties); depth of lamina propria invasion and microscopic vascular invasion are also factors predictive of understaging.

High grade NMIBC can be treated with TURBT and intravesical chemo- immunotherapy, but this treatment is absolutely insufficient if there is an error in stage determination, that can lead to a delay in radical surgery and 
worse prognosis. Defining the exact stage of bladder tumor is essential for planning appropriate treatment and determining the patient's prognosis. In this study we have considered a selective group of patients with highgrade bladder tumor, who were staged T1 at first TURBT; the second TURBT was not performed in our patients, since the bladder was extensively affected by the tumor, and residual tumor was present in $76.14 \%$ of cases.

According to our observation, when evaluating patients with NMIBC at first TURBT, we believe that it is important to consider the following factors that are able to increase the risk of understaging: high grade tumor, deep lamina propria and microscopic vascular invasion, multiple tumors, tumor size $>6 \mathrm{~cm}$, tumor location (trigone and dome), presence of residual tumor.

When these risk factors are present, we suggest to examine carefully the case and consider whether avoiding delays with performing a second TURBT and proceed straight to radical cystectomy could be a possible option; in this situation, performing an early cystectomy could lower the risk of worse pathological finding due to rapid disease progression of the high grade tumors, and can prolong survival.

\section{REFERENCES}

1. Ploeg M, Aben KK, Kiemeney LA. The present and future burden of urinary bladder cancer in the world. World J Urol. 2009; 27:28993.

2. Babjuk M, Burger M, Zigeuner R, et al. EAU guidelines on nonmuscle-invasive urothelial carcinoma of the bladder: update 2013. Eur Urol. 2013; 64:639-53.

3. Ramírez-Backhaus M, Domínguez-Escrig J, Collado A, et al. Restaging Transurethral Resection of Bladder Tumor for High-risk Stage Ta and T1 Bladder Cancer. Curr Urol Rep. 2012; 13:109-14.

4. Poulsen AL, Horn T, Steven K. Radical cystectomy: extending the limits of pelvic lymph node dissection improves survival for patients with bladder cancer confined to the bladder wall. J Urol. 1998; 160:2015-9.

5. Edge SB, Compton CC. The American Joint Committee on Cancer: the 7th edition of the AJCC cancer staging manual and the future of TNM. Ann Surg Oncol. 2010; 17:1471-4.

6. Sauter G, Algaba F, Amin M, et al. Tumours of the urinary system: non-invasive urothelial neoplasias. In: Eble JN, Sauter G, Epstein Jl, Sesterhenn I, eds. WHO classification of classification of tumours of the urinary system and male genital organs. Lyon: IARCC Press, 2004, pp. 29-34.
7. Kirkali Z, Chan T, Manoharan M, et al. Bladder cancer: epidemiology, staging and grading, and diagnosis. Urology. 2005; 66:4-34.

8. Ark JT, Keegan KA, Barocas DA, et al. Incidence and predictors of understaging in patients with clinical T1 urothelial carcinoma undergoing radical cystectomy. BJU Int. 2014; 113:894-9.

9. Nieder AM, Brausi M, Lamm D, et al. Management of stage T1 tumors of the bladder: International Consensus Panel. Urology. 2005; 66:108-25.

10. Dutta SC, Smith JA, Shappell SB, et al. Clinical under staging of high risk nonmuscle invasive urothelial carcinoma treated with radical cystectomy. J Urol. 2001; 166:490-3.

11. Hall MC, Chang SS, Dalbagni G, et al. Guideline for the management of nonmuscle invasive bladder cancer (stages Ta, T1, and Tis): 2007 update. J Urol. 2007; 178:2314-30.

12. Montie JE, Clark PE, Eisenberger MA, et al. National Comprehensive Cancer Networkl. Bladder cancer. J Natl Compr Canc Netw. 2009; 7:8-39.

13. McLaughlin S, Shephard J, Wallen E, et al. Comparison of the Clinical and Pathologic Staging in Patients Undergoing Radical Cystectomy for Bladder Cancer. International Braz J Urol. 2007; 33:25-32.

14. Dalbagni G, Herr HW, Reuter VE: Impact of a second transurethral resection on the staging of $\mathrm{T} 1$ bladder cancer. Urology. 2002; 60:822-4.

15. Chang SS, Hassan JM, Cookson MS, et al. Delaying radical cystectomy for muscle invasive bladder cancer results in worse pathological stage. J Urol. 2003; 170:1085-7.

16. Sanchez-Ortiz RF, Huang WC, Mick R, et al. An interval longer than 12 weeks between the diagnosis of muscle invasion and cystectomy is associated with worse outcome in bladder carcinoma. J Urol. 2003; 169:110-5.

17. Mariappan P, Zachou A, Grigor KM. Detrusor muscle in the first, apparently complete transurethral resection of bladder tumour specimen is a surrogate marker of resection quality, predicts risk of early recurrence, and is dependent on operator experience. Eur Urol. 2010; 57:843-9.

18. Zurkirchen MA, Sulser T, Gaspert A, Hauri D. Second transurethral resection of superficial transitional cell carcinoma of the bladder: a must even for experienced urologists. Urol Int. 2004; 72:99-102.

19. Vasdev N, Dominguez-Escrig J, Paez E, et al. The impact of early re-resection in patients with $p T 1$ high-grade non-muscle invasive bladder cancer. Ecancermedicalscience. 2012; 6:269.

20. Solsona E, Iborra I, Rubio J, Casanova J, Almenar S. The optimum timing of radical cystectomy for patients with recurrent highrisk superficial bladder tumour. BJU Int. 2004; 94:1258-62.

\section{Correspondence}

Daniele Minardi, MD

d.minardi@gostec.net

Giulio Milanese, MD

Gianni Parri, MD

Vito Lacetera, MD

Giovanni Muzzonigro, MD

Clinica Urologica, Università Politecnica delle Marche, A.O. Ospedali Riuniti Via Conca 71 - 60020 Ancona, Italy 